\title{
Membrane Formation via Thermally-Induced Phase Separation from Polypropylene/Fatty Acids Systems
}

\section{Jae-Jin Kim*1, Jeong Rim Hwang, Un Young Kim, and Sung Soo Kim*2}

\author{
Membrane Laboratory, The Korea Institute of Science and Technology \\ P. O. Box 131, Cheongryang, Seoul, Korea \\ ${ }^{* 1}$ To whom all correspondences should be addressed. \\ *2Dept. of Chemical Engineering, Kyung Hee University, Seoul, Korea
}

Microporous polypropylene membranes were prepared via thermally-induced phase separation from mixtures of polypropylene/soybean oil, polypropylene/oleic acid, and polypropylene/linoleic acid. The phase diagram of each system was obtained by thermal analysis and cloud point measurements. It was proved that each system has a liquid-liquid phase separation region at concentrations less than the monotectic composition. A cellular structure was obtained for each system, which is the typical structure for liquid-liquid phase separation via slow cooling. Fine structure with tiny polypropylene spherulites was obtained via quenching process. Mobility of diluent was related with its rejection during the polypropylene spherulite formation. A melt spinning apparatus was designed to produce microporous hollow fiber membranes. The mechanical strength of the hollow fiber seemed to be related to solution viscosity. Polypropylene/soybean oil samples had much greater solution viscosity than the other systems, and formed fibril structures with enhanced mechanical strength.

Key words : microporous membranes, membrane formation and structure, phase separation, thermally-induced, hollow fiber, melt spinning

\section{Introduction}

The thermally-induced phase separation (TIPS) process has been utilized for making microporous membranes ${ }^{1 \sim 3)}$. Various polymers and diluents have been used for the TIPS process, and some of them have been commercialized ${ }^{1 \sim 4)}$. Polypropylene (PP) is one of the most popular TIPS membrane materials because of its many advantages over other polymers ${ }^{4)}$. The choice of diluent is important since it determines the interactions with the polymer and consequently the phase separation mechanism ${ }^{1 \sim 7)}$. $\mathrm{PP} /$ mineral oil undergoes solid-liquid phase separation, which results in a spherulitic structure via PP crystallization ${ }^{8)}$. PP/ n, n-bis (2-hydroxyethyl) tallowamine (TA) undergoes liquid-liquid phade separation when the initial composition is less than the monotectic composition $^{2.4 .9)}$. Liquid-liquid phase separation can result in a cellular structure via nucleation and growth or coarsening after spinodal decomposition 4. 10.11).

A cellular structure with connecting pores is useful for ultrafiltration and microfiltration membranes. Ultrafiltration and microfiltration processes are often used in biological processes, medical divices, and food processes ; therefore, the toxicity of the diluent as well as the polymer is critical. Soybean oil is an appropriate candidate for these purposes, since PP/soybean oil system 
Table 1. Constituents of soybean oil

\begin{tabular}{|l|c|}
\hline Constituents & Composition (\%) \\
\hline Linoleic acid & 50.7 \\
Oleic acid & 28.9 \\
Palmitic acid & 9.8 \\
Linoleic acid & 6.5 \\
Stearic acid & 2.4 \\
Arachidic acid & 0.9 \\
Palmitoleic acid & 0.4 \\
Lauric acid & 0.2 \\
Myristic acid & 0.1 \\
Others & 0.1 \\
\hline
\end{tabular}

undergoes liquid-liquid phase separation and the soybean oil has little toxicity. Moreover, soybean oil is available at cheaper price than previously reported diluents for PP.

Flat membranes were fabricated on a hot stage apparatus and hollow fiber membranes were made via melt spinning process from the PP/ soybean oil mixture. Pure oleic acid and linoleic acid, the constituents of soybean oil, were also used as diluents. Thermal analysis and cloud point measurement were performed for each $\mathrm{PP} /$ diluent system to help understand the phase separation mechanism and the resulting structure.

\section{Materials and Methods}

PP was supplied by Daehan Yuhwa Co., Korea, and its weight average molecular weight was 400,000 , and melt index was $2 \mathrm{~g} / 10 \mathrm{~min}$. Soybean oil was purchased from Cheil Food Chemicals Co., Korea, and its constituents are listed in Table 1. Oleic acid was purchased from Kanto Chemical Co., Japan, and linoleic acid from Sigma Chemical Co., Trichlorotrifluoroethane (Freon 113) was from Ulsan Chemical Co., Korea. A Perkin Elmer DSC 4 was used for the thermal analyses. A Mettler FP 82 hot stage and FP 80 central processor were used for flat membrane fabrication and cloud point measurement. A vessel extrusion apparatus was manufactured for hol-

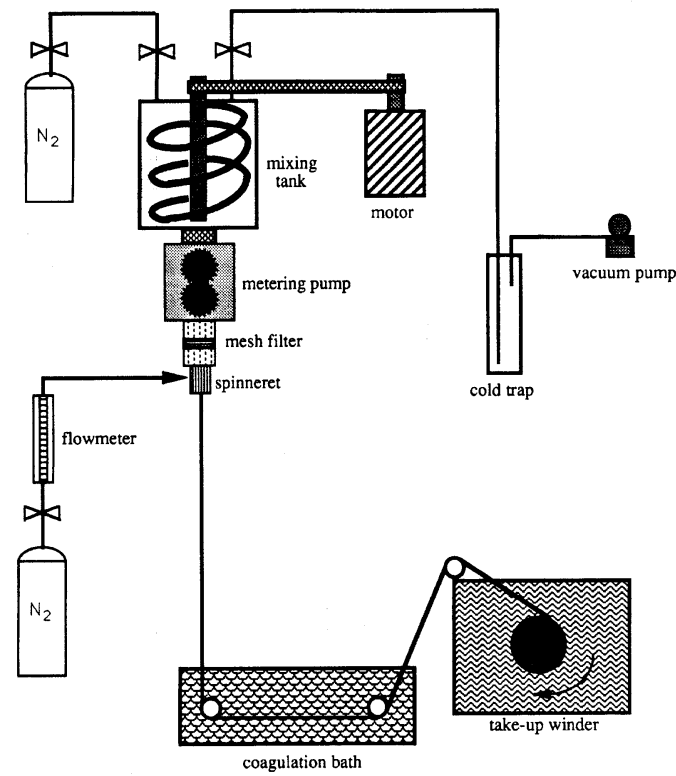

Fig. 1 A schematic diagram of vessel extrusion process.

low fiber spinning. The structure of each membrane was examined by using a scanning electron microscope (Hitachi S-510). A Ubbelohde capillary viscometer of diameter $1.13 \mathrm{~mm}$ was used to measure the efflux times of pure diluents. The tensile strength of each hollow fiber was measured by Instron (Model \#4201) using a load cell of $5 \mathrm{Kg}$ at a crosshead speed of $10 \mathrm{~cm} / \mathrm{min}$. Melt index of each $\mathrm{PP} /$ diluent mixture was measured using an apparatus made by Nihon Rigaku Kogyo Co. (Model \#204-65 T) with the total load of $2.16 \mathrm{Kg}$.

\section{Fabrication of Flat Membranes}

Measured amounts of PP and diluent were mixed in a test tube and purged with nitrogen. The test tube was flame sealed and placed in an oven preset at $200^{\circ} \mathrm{C}$ for 24 hours to make a homogeneous melt solution. The test tube was quenched in ambient water to recover the homogeneous sample. The chopped sample was placed between a pair of microscope cover slips sealed with teflon tape, placed on a hot stage apparatus preset at $200^{\circ} \mathrm{C}$ 


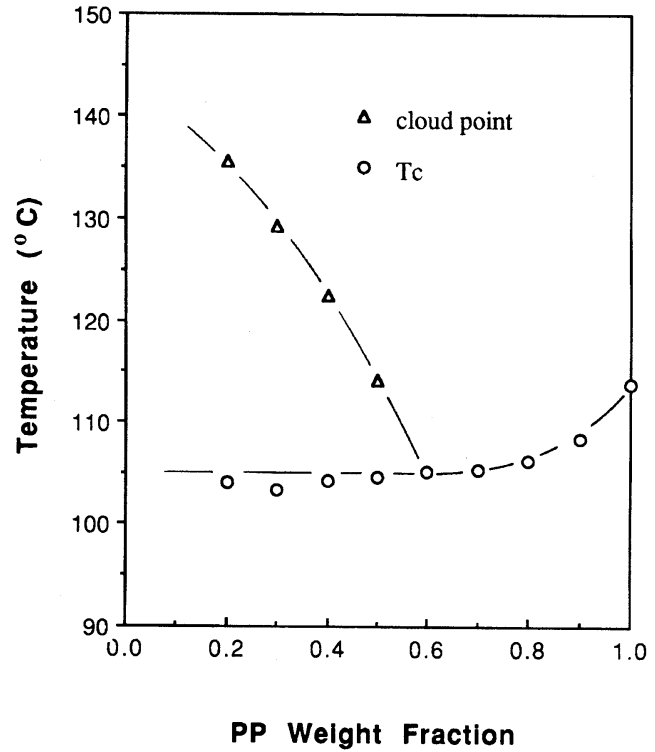

Fig. 2 Phase diagrams of $\mathrm{PP} /$ soybean oil system determined at $10^{\circ} \mathrm{C} / \mathrm{min}$ cooling.

for 3 minutes, and cooled at a rate of $10^{\circ} \mathrm{C} / \mathrm{min}$ or quenched to $25^{\circ} \mathrm{C}$. The film sample was peeled from the cover slips and immersed in Freon 113 for diluent extraction. The sample was dried in a vacuum oven to make a flat TIPS membrane.

\section{Melt Spinning of Hollow Fiber Membranes}

Hollow fibers were prepared via melt spinning. Difficulties in premixing of $\mathrm{PP}$ and the liquid diluents and feeding through the hopper prevented use of a conventional extruder. Therefore, vessel extrusion apparatus was designed as shown in Figure 1. Measured amounts of PP and diluent were placed in the vessel and heated to $220^{\circ} \mathrm{C}$ under the nitrogen purged atmosphere. The spiral mixing blade was employed to enhance the sample homogeneity, and the mixing time was 4 hours. Under the nitrogen pressure the metered amount of homogeneous melt was fed to a spinneret by a gear pump. A spinneret of tube-in-orifice type was manufactured; its inner diameter was $3 \mathrm{~mm}$

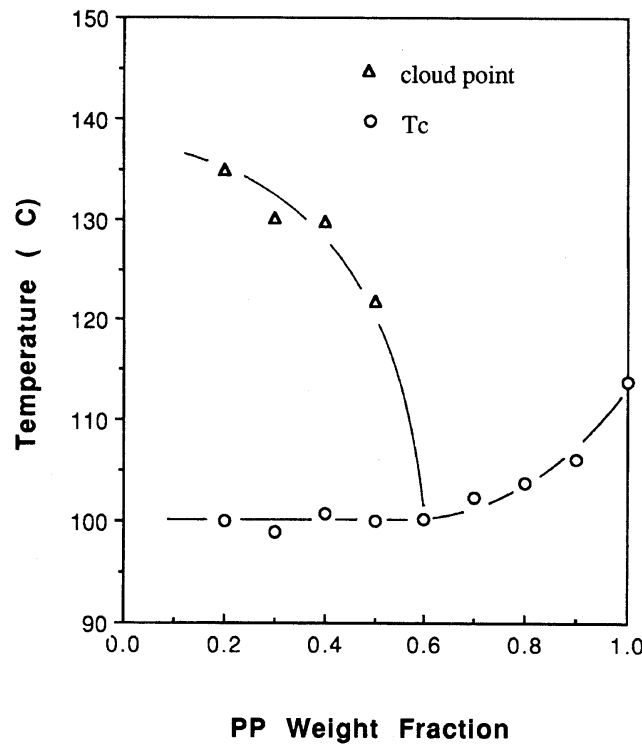

Fig. 3 Phase diagrams of $\mathrm{PP} /$ oleic acid system determined at $10^{\circ} \mathrm{C} / \mathrm{min}$ cooling.

and outer diameter was $5 \mathrm{~mm}$. Another stream of nitrogen was introduced into the spinneret to make a lumen at the center of the fiber. The spinneret temperature was varied from 165 to $185^{\circ} \mathrm{C}$ according to the composition and the molecular weight of PP.

The hollow fiber melt was partly cooled in the air, and it entered the coagulation bath where it was completely solidified and stretched. The Freon 113 in the coagulation bath was used as a diluent extractant as well as a coagulant, and diluent at the outer surface of the fiber was partly extracted while it passed through the coagulation bath. The hollow fiber was wound on a take-up winder at a specified melt-draw ratio. The remaining diluent was completely extracted in Freon 113 for 24 hours. Finally, it was dried in a vacuum oven for the removal of the remaining solvent and diluent.

\section{Phase Diagrams}

The crystallization temperatures of the PP/ soybean oil, PP/oleic acid, and PP/linoleic acid 


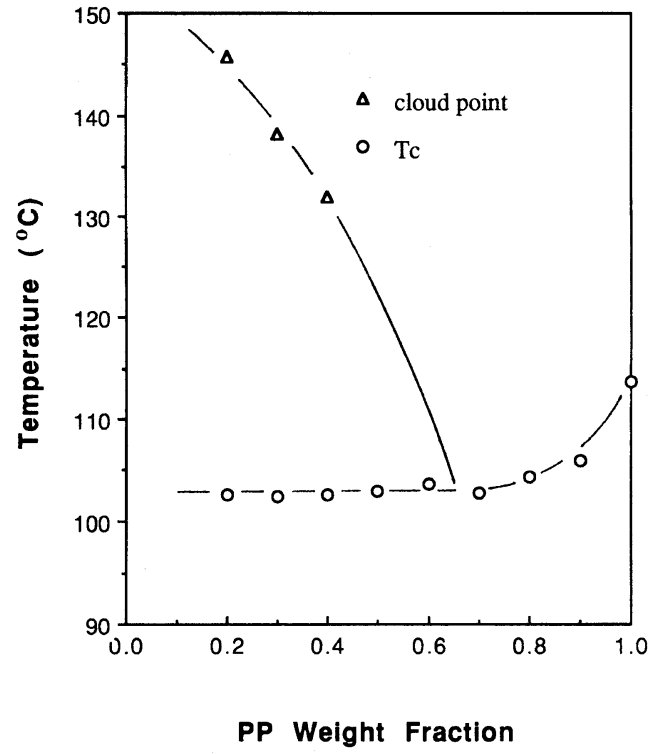

Fig. 4 Phase diagrams of $\mathrm{PP} /$ linoleic acid system determined at $10^{\circ} \mathrm{C} / \mathrm{min}$ cooling.

systems were measured by DSC at a cooling rate of $10^{\circ} \mathrm{C} / \mathrm{min}$. As shown in Figures 2,3, and 4, each phase diagram has a flat crystallization curve at concentration less than the monotectic composition, which is a characteristic of liquid-liquid phase separation. The monotectic point of the $\mathrm{PP} /$ soybean oil system is located around $60 \mathrm{wt}$. $\%$ of $\mathrm{PP}$ and $105^{\circ} \mathrm{C}$ at a cooling rate of $10^{\circ} \mathrm{C} / \mathrm{min}$, 60 wt. \% of PP and $100^{\circ} \mathrm{C}$ for the PP/oleic acid system, and 70 wt. $\%$ of $\mathrm{PP}$ and $103.5^{\circ} \mathrm{C}$ for the PP/ linoleic acid system.

\section{Structure Analysis}

$\mathrm{PP} /$ soybean oil, $\mathrm{PP} /$ oleic acid, and PP/linoleic acid samples were prepared in flat form with $\mathrm{PP}$ concentration of $40 \mathrm{wt} . \%$. They were cooled at a rate of $10^{\circ} \mathrm{C} / \mathrm{min}$ or directly quenched to $25^{\circ} \mathrm{C}$. Since 40 wt.\% of PP is less than the monotectic concentration, each slowly cooled sample underwent liquid-liquid phase separation followed by PP crystallization after it reaches the monotectic temperature. Therefore, a cellular structure was
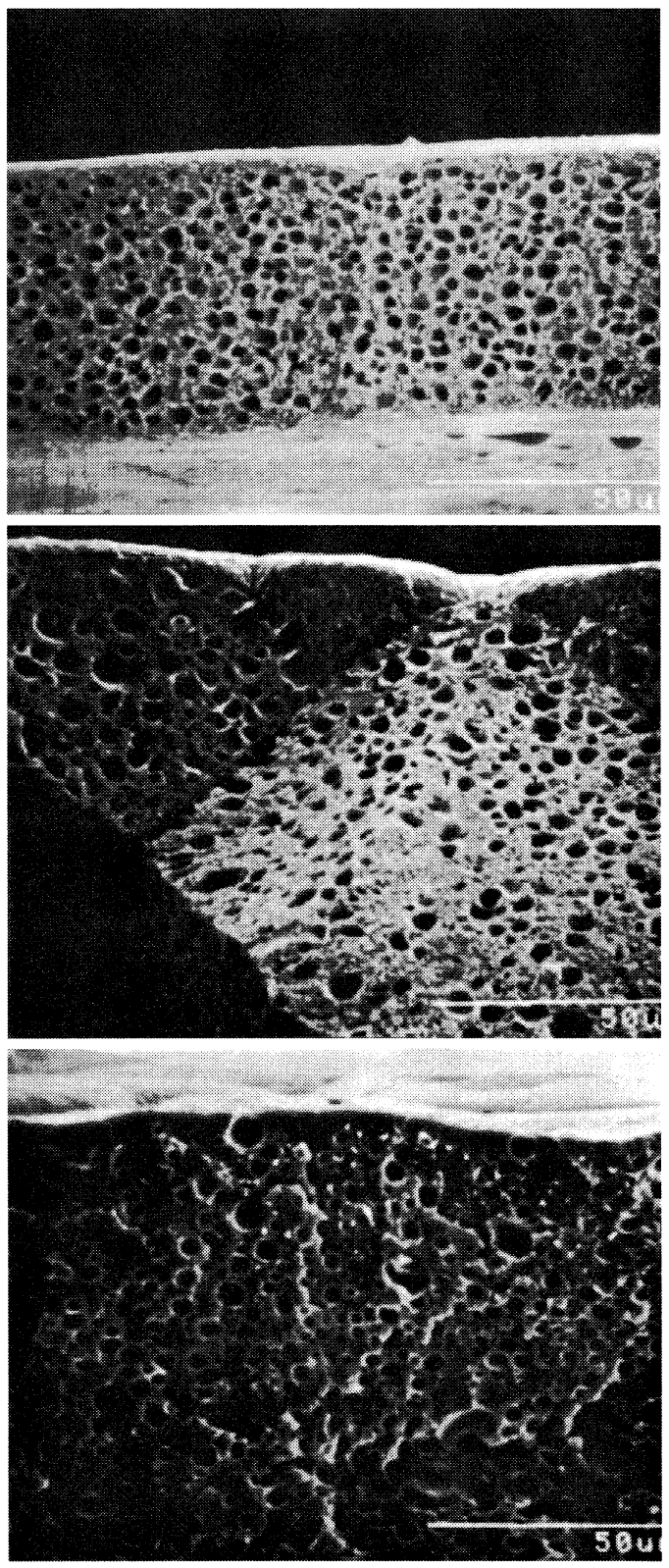

Fig. 5 Cross sectional views of flat membranes cooled at rate of $10^{\circ} \mathrm{C} / \mathrm{min}$ from $200^{\circ} \mathrm{C}$ to $25^{\circ} \mathrm{C}$ (top : $\mathrm{PP} /$ soybean oil, middle : PP/oleic acid, bottom : $\mathrm{PP} /$ linoleic acid).

obtained for every case as shown in Figure 5. The cellular structure was obtained via nucleation and growth or coarsening after spinodal decomposition, and it is a characteristic structure of liquid- 

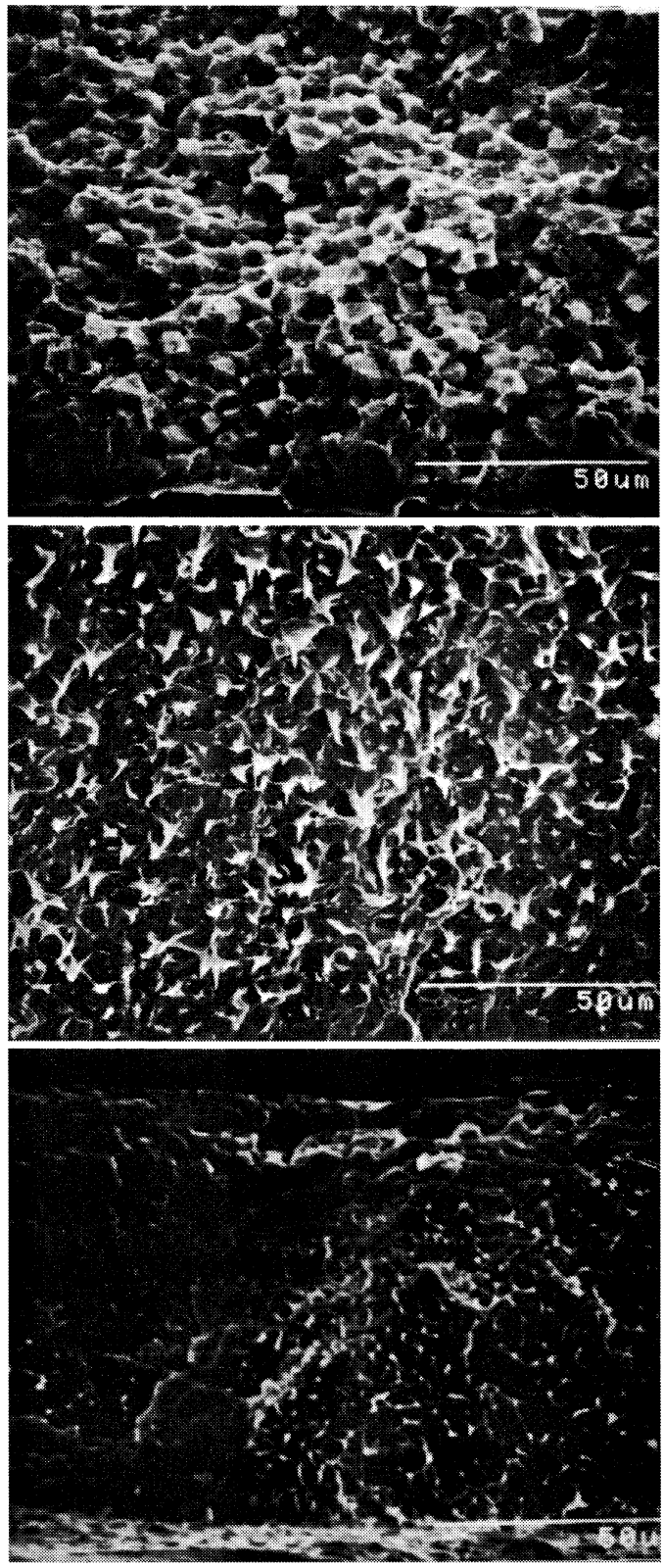

Fig. 6 Cross sectional views of flat membranes quenched from $200^{\circ} \mathrm{C}$ to $25^{\circ} \mathrm{C}$ (top : $\mathrm{PP} /$ soybean oil, middle : $\mathrm{PP} /$ oleic acid, bottom : $\mathrm{PP} /$ linoleic acid).

liquid phase separation ${ }^{10 \sim 12)}$.

When the same sample were directly quenched from $200^{\circ} \mathrm{C}$ to $25^{\circ} \mathrm{C}$ water, they formed a fine structure with tiny PP spherulites as shown in Figure

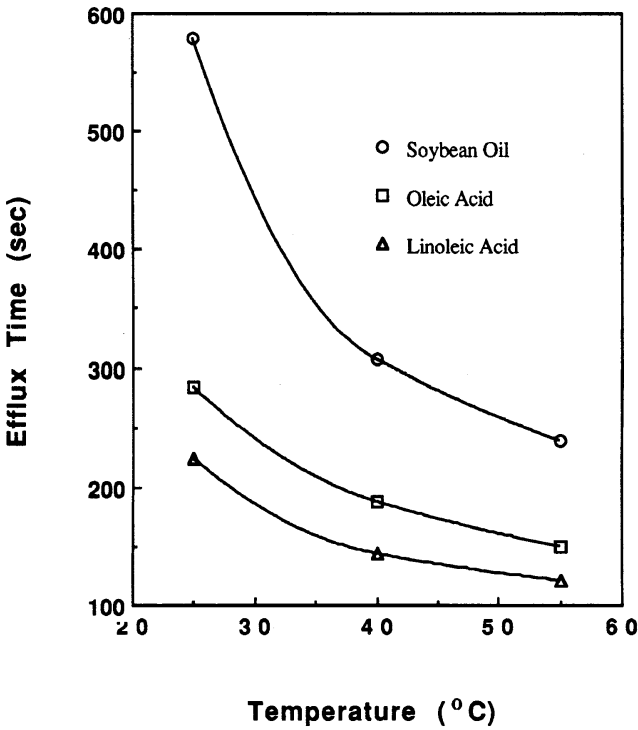

Fig. 7 Efflux time of each diluent through Ubbelohde type capillary viscometer.

6. Since every sample was under the thermodynamic conditions of liquid-liquid phase separation and PP crystallization, simultaneous liquid-liquid and solid-liquid phase separations could be expected for every sample. The PP/soybean oil sample formed tiny spherulites with micropores within the spherulites, while PP/oleic acid and PP/ linoleic acid samples have dense PP spherulites free of such tiny micropores. Micropores within the tiny spherulite are frequently observed in solid-liquid phase separation systems ${ }^{4,8)}$. The micropores result from unrejected diluent due to its low mobility ${ }^{13)}$. Diluent mobility was indirectly measured by measuring the efflux time of each pure diluent through a Ubbelohde capillary viscometer over a temperature range of 25 to $55^{\circ} \mathrm{C}$. Soybean oil has greater efflux time than the other diluents used in this study as shown in Figure 7. Due to the experimental difficulties in bath temperature control, efflux times at the TIPS temperauture were not measured. However, trend can be extrapolated to TIPS temperature. Soybean oil was less rejected from the growth front of the PP spherulites than oleic acid and linoleic acid due to 
Table 2. Spinning conditions for hollow fiber membrane formation

\begin{tabular}{|l|l|}
\hline Coagulant & Freon 113 \\
Vessel Temperature & $220^{\circ} \mathrm{C}$ \\
Spinning Temperature & $170^{\circ} \mathrm{C}$ \\
Spinning Speed & $8.0 \mathrm{~cm} / \mathrm{min}$ \\
Nitrogen Flow Rate & $3 \mathrm{~cm}^{3} / \mathrm{min}$ \\
Distance between spinneret and & $40 \mathrm{~cm}$ \\
coagulation bath & 1000 \\
Melt-draw ratio & \\
\hline
\end{tabular}

its low nobility.

The hollow fibers were prepared from $\mathrm{PP} /$ soybean oil, PP/oleic acid, and PP/linoleic acid samples (30 wt. $\%$ of PP) with the spinning conditions listed in Table 2. As shown in Figure 8, each sample formed a microporous structure. When the structures of the hollow fiber membranes were compared with those of the quenched flat membranes (Figure 6), the former has more porous structure than the latter. The difference in porous structure came from two factors ; axial stretching of the hollow fibers and immediate contact with extracting solvent before the completion of the phase separation.

For flat membrane fabrication, there was no shear stress for stretching, whereas the spun hollow fiber was stretched at a melt-draw ratio specified in Table 2. Therefore, more pores could be formed via axial stretching of hollow fiber membranes. The melt-draw ratio is defined as the ratio of take-up speed to spinning speed. More details of melt-draw ratio effect are discussed elsewhere ${ }^{14,15)}$. The phase separation and solidification of flat membranes were completed without any contact with extracting solvent, and extraction of diluent was performed after the completion of structure formation via TIPS. The hollow fiber melt contacted extracting solvent before the completion of phase separation and solidification of the melt. Three processes occurred in the bath ; phase separation of the sample by cooling, extraction of the diluent, and phase separation via counter diffusion of diluent and solvent. These

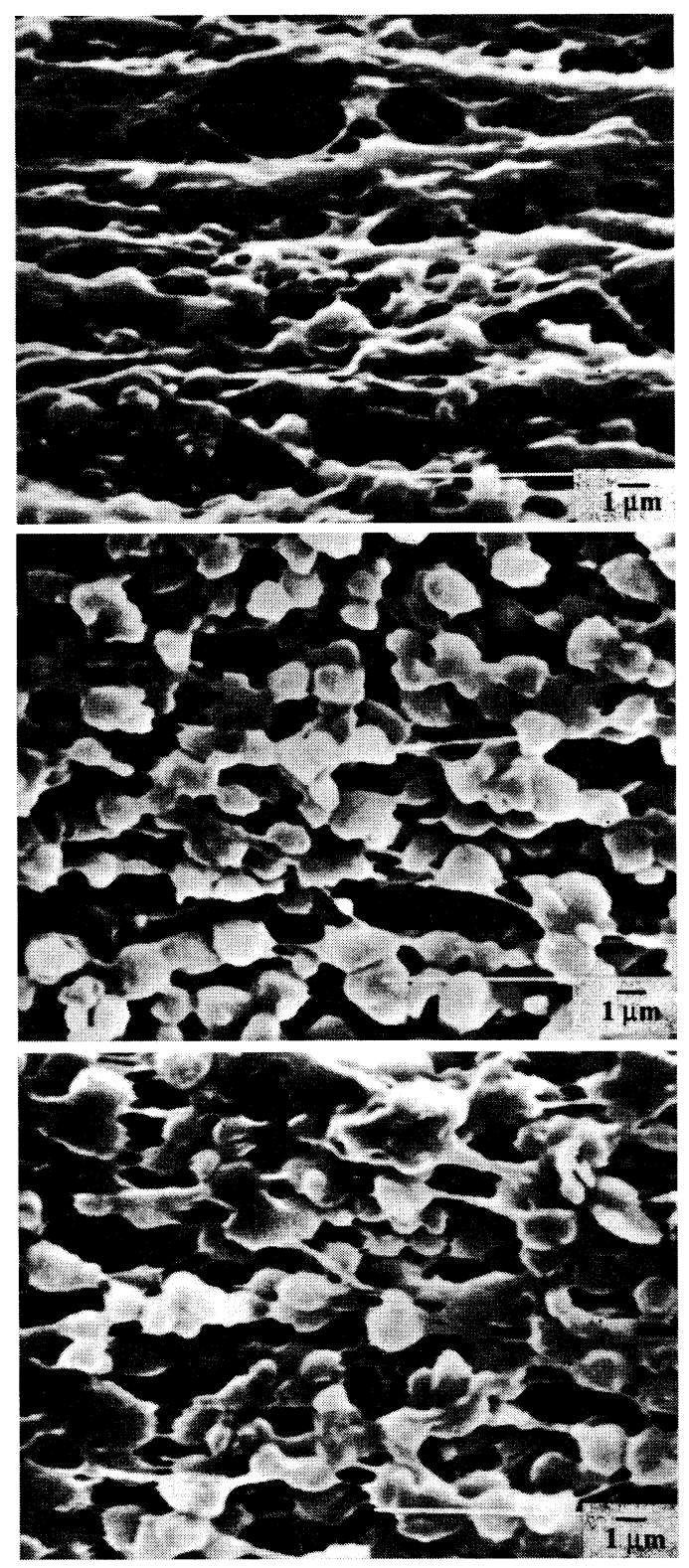

Fig. 8 Microporous structures of hollow fiber membranes

(top : PP/soybean oil, middle : PP/ oleic acid, bottom : PP/linoleic acid).

factors cause the structures of the hollow fiber membranes to be different from those of the flat membranes.

The mechanical strength of the hollow fiber made from $\mathrm{PP} /$ soybean oil mixture was much 
Table 3. Melt index of each PP/diluent mixture ( 30 wt. \% of PP) at $190^{\circ} \mathrm{C}$ and tensile strength of each hollow fiber

\begin{tabular}{|l|c|c|c|}
\hline \multicolumn{1}{|c|}{ Sample } & PP/soybean oil & PP/oleic acid & PP/linoleic acid \\
\hline Melt index* $(\mathrm{g} / 10 \mathrm{~min})$ & 19.6 & 44.5 & 46.3 \\
Tensile Strength** $\left(\mathrm{Kg}_{f} / \mathrm{mm}^{2}\right)$ & 2.64 & 1.56 & 0.95 \\
\hline
\end{tabular}

${ }^{*}$ Melt index of $\mathrm{PP} /$ diluent mixture at $190^{\circ} \mathrm{C} \quad$ Total load : $2.16 \mathrm{Kg}$

**Tensile strength of hollow fiber after removal of diluent Local cell : $5 \mathrm{Kg}$, Crosshead speed : $10 \mathrm{~cm} / \mathrm{min}$

higher than those from $\mathrm{PP} /$ oleic acid and $\mathrm{PP} /$ linoleic acid mixtures as shown in Table 3. The difference can be explained in terms of the fibril structure of the fibers. As shown in Figure 8, there were many fibrils in the hollow fibers made from the $\mathrm{PP} /$ soybean oil mixture, and those from $\mathrm{PP} /$ oleic acid and PP/linoleic acid mixtures have tiny isolated PP spherulites without connecting fibrils. The presence of the fibrils between the PP spherulites enhanced the mechanical strength of the fiber. Formation of the fibrils is related to the viscosity of the PP/diluent melt. During the solidification, the hollow fiber melt can be stretched in the spinning direction with appropriate melt viscosity to make fibrils. However, the fibrils cannot resist the shear stress in the spinning direction if the melt viscosity is low.

The melt index of each $\mathrm{PP} /$ diluent mixture (30 wt.\% PP) was measured according to ASTM D1238-88 to show that the melt indices of the PP/ oleic acid and PP/linoleic acid sample were much higher than that of $\mathrm{PP} /$ soybean oil sample at $190^{\circ} \mathrm{C}$ (Table 3 ). Therefore, low melt viscosities of $\mathrm{PP} /$ oleic acid and PP/linoleic acid samples failed to form the fibril structures to result in the poor mechanical strengths. $\mathrm{PP} /$ soybean oil system is more recommendable for making microporous hollow fiber membranes than the other $\mathrm{PP} /$ diluent systems reported here.

\section{Conclusions}

Microporous structures for ultrafiltration and microfiltration were obtained from $\mathrm{PP} /$ soybean oil, PP/oleic acid, and PP/linoleic acid systems in both flat and hollw fiber types. Thermal analysis and cloud point measurement proved that these systems have liquid-liquid phase separation behavior, and the location of binodal region is much related to the diluent polarity. A cellular structure was obtained for each system at a cooling rate of $10^{\circ} \mathrm{C} / \mathrm{min}$ confirming the liqued-liqued phase separation. Diluent mobility determined the size of the micropores within the PP spherulites. Stretching of the hollow fibers by melt-drawing enhanced the porosity of the membranes. Simultaneous phase separation and diluent extraction also affected the structure of the membranes. Melt viscosity influences the formation of fibrils between the spherulites and the mechanical strength of the hollow fiber.

\section{References}

1) A. J. Castro, Method for making microporous products, U.S. Patent 4,247, 498, 1980.

2) G. H. Shipmam, Microporous sheet materials, method of making and articles made therewith, U. S. Patent, 4,539, 256, 1985.

3) D. R. Lloyd, J. W. Barlow and K. E. Kinzer, Microporous membrane formation via thermally-induced phase separation, in $\mathrm{K}$. K. Sirkar and D. R. Lloyd (Eds.), New Membrane Materials and Processes for Separation, AIChE Symp. Ser, No. 261, American Institute of Chemical Engineers, New York, NY, 1988.

4) S. S. Kim and D. R. Lloyd, Microporous membrane formation via thermally-induced phase separation. III. Effect of thermodynamic interactions on the structure of isotactic polypropylene membranes J. Membrane Sci, 64 (1991) 13. 
5) S. S. Kim and D. R. Lloyd, Thermodynamics of polymer-diluent systems for thermally-induced phase separation I. Determination of equation of state parameters, Polymer, 33 (1992) 1026.

6) S. S. Kim and D. R. Lloyd, Thermodynamics of polymer-diluent systems for thermallyinduced phase separation. П. Solid-liquid phase separation systems, Polymer, 33 (1992) 1036.

7) S. S. Kim and D. R. Lloyd, Thermodynamics of polymer-diluent systems for thermallyinduced phase separation. III. Liquid-liquid phase separation systems, Polymer, 33 (1992) 1047.

8) D. R. Lloyd, K. E. Kinzer and H. S. Tseng, Microporous membrane formation via thermally-induced phase separation. I. Solidliquid phase separation, J. Membrane Sci., 52 (1990) 239.

9) W. C. Hiatt, G. H, Vitzthum, K. B. Wagener, K. Gerlach, and C. Josefiak, Microporous membranes via upper critical temperature phase separation, in D. R. Lloyd (Ed.), Materials Science of Synthetic Membranes, ACS Symposium Series No. 269, American Chemical Society, Washington, DC, 1985.

10) G. T. Caneba and D. S. Soong, Polymer membrane formation through the thermal-inversion process. 1. Experimental Study of mem-
膜 (MEMBRANE), Vol. 19 No. 4 (1994)

brane structure Macromolecules, 18 (1985) 2538.

11) G. T. Caneba and D. S. Soong, Polymer membrane formation through the thermal-inversion process. 2. Mathematical modeling of the membrane structure formation, Macromolecules, 18 (1985) 2545.

12) D. R. Lloyd, S. S. Kim and K. E. Kinzer, Microporous membrane formation via thermally-induced phase separation. II. Liquidliquid phase separation, J. Membrane Sci, 64 (1991) 1 .

13) S. S. Kim, G. B. A. Lim, A. A. Alwattari, Y. F. Wang and D. R. Lloyd, Microporous membrane formation via thermally-induced phase separation. V. Effect of diluent mobility and crystallization on the structure of isotactic polypropylene membranes, J. Membrane Sci, 64 (1991) 41.

14) J. J. Kim T. S. Jang, Y. D. Kwon, S. S. Kim and U. Y. Kim, Structural study of microporous polypropylene hollow fiber membranes made via melt spinning and cold-stretching method, J.Membrane Sci, in press (1994).

15) J. J. Kim, J. R. Hwang, U. Y. Kim and S. S. Kim, Operation parameters of melt spinning of polypropylene hollow fiber membranes, submitted to J. Membrane Sci, (1994).

（受付 1993 年 8 月 10 日) 